\title{
Research and Application of PDM and CAD Integration Technology
}

\author{
Jiu Sun ${ }^{1, a}$ and Yuemiao Wang $^{2, b}$
}

${ }^{1}$ Department of Experimental Teaching, Yancheng Institute of Technology, Yancheng, China

${ }^{2}$ School of Mechanical Engineering, Nanjing University of Science and Technology, Nanjing, China

asunjiu@ycit.cn, b524057192@qq.com

Keywords: Product data management, Application software Integration, Pro/e, Teamcenter

\begin{abstract}
In the informationization construction process of mechanical manufacturing industry, CAX application software is widely used in the process of the design and manufacture in enterprise, which significantly improves the efficiency and reliability of production design. The research mainly discusses Product data management (PDM) and CAD integration technology, puts forward the PDM system integration framework and PDM and CAD two-way integrated structure, and makes further research to Pro/E and Teamcenter integration. Teamcenter software is the information transfer bridge of enterprise as an integrated platform. The integration between Teamcenter and Pro/E system has very important significance to simplify the interactive operation.
\end{abstract}

\section{Introduction}

With the development of enterprise informatization process, application software used by enterprise is more and more. The width and depth of the information integration has also changed from the initial information integration development to today's process integration (such as concurrent engineering), and requirements further integration among enterprises (such as agile manufacturing, etc.). Along with the PDM system (product data management system) introduction, the user, on the one hand, in the hope that it can realize all kinds of software to generate data and documentation for effective management. On the other hand, in the PDM environment, it also hopes to realize information sharing between users, collaborative work, application system and PDM system in the consistency of the data objects and design among application software. These are required to change traditional method based on the database to realize system integration in the past into application integration based on product data management (PDM). Integration is to make the resources and application based on the information technology gather into a collaborative work whole, and it contains three aspects of management and control which are function interaction, information sharing and data communication [1]. In PDM, integration is to make the people, production management system and engineering technology system combine to form a unified whole, and make whole enterprise within the scope of the workflow, logistics and information flow keep unobstructed and mutual organic contact. Therefore, in product data management system development and implementation, it needs to research application tool integration technology.

As computer generates a lot of electronic document, most of the enterprise is to use shared directory structure to manage the electronic document simply. Design is in the first place of enterprise production, and CAD and subsequent management information system integration is of particularly important significance. It can form a continuous flow of information by realizing the integration, and to further improve the application of CAD efficiency, to improve overall enterprise's operation efficiency. Therefore, how to ensure the integrity of the electronic document, consistency and accuracy is a big problem after the application of CAD [2]. With the application of PDM in manufacturing system, the integration of PDM and CAD technology gets fast development. As the enterprise information integration management platform, PDM supports CAD product design process and its output materials, at the same time CAD also need to meet the many demands of PDM and provide support to the PDM product attribute database.

Research is based on Teamcenter software platform of Siemens PLM Software Company and its Pro/E software platform of the United States parameter technology company (PTC). The aim of the 
application of the integration of two softwares is to manage the CAD data in enterprise. According to the needs of the enterprises, the environmental configuration and integrated implementation and application are convenient to manage data for enterprise. According to the actual demand of the Pro/E and Pro/E integration for enterprise, it can combine with CAD integrated implementation in some PDM project [3]. Some of the actual application can solve Teamcenter and Pro/E integrated installation, configuration and interface function application problems in the implementation of enterprise.

\section{The PDM and CAD integration framework}

PDM system carries through data exchange and integration through the integrated interface and each application and management system. PDM system integrates all related product data and makes it stored in the PDM system database. PDM system is responsible for managing all data and exchanging information with other system. In the integrated framework, PDM system can be unified to manage and product all the pertinent information related to reallife cycle. PDM system integration framework is shown in fig.1.

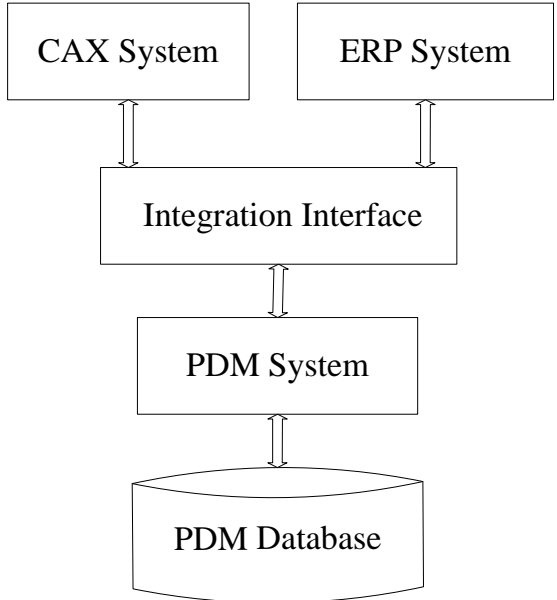

Fig.1 PDM system integration framework

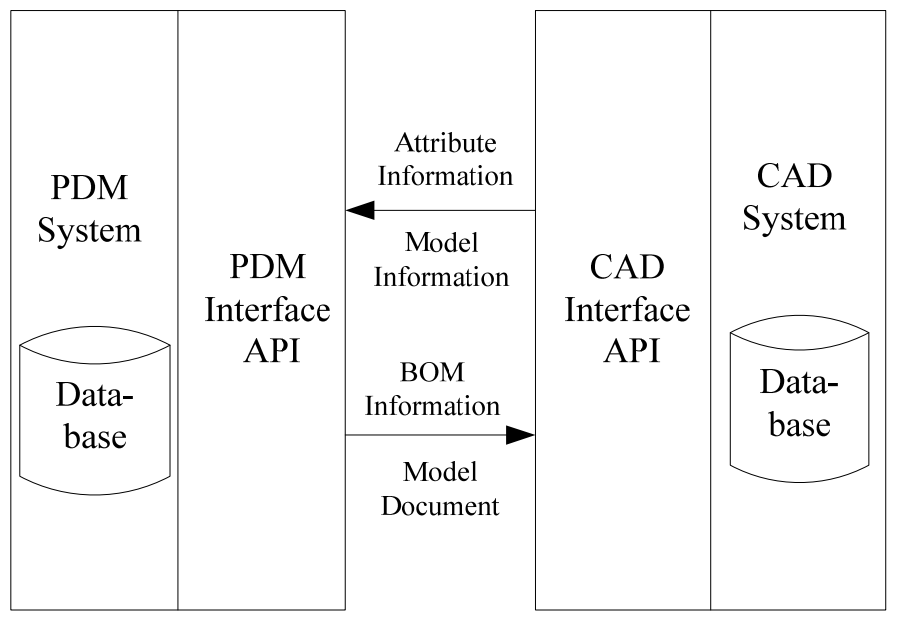

Fig.2 PDM and CAD two-way integrated structure

CAD, CAPP and CAM, ERP system can't exchange of information with each other directly, and their information transfer has become information exchange with PDM system respectively. CAD, CAPP and CAM, ERP system picks up the respective needed information from PDM system through the interface provided by PDM system. Their application result also is put back to the PDM system database, so as to realize the integration of enterprise management system.

The data exchange of bidirectional integration between PDM and CAD mainly include the next two kinds of information, and they are product structure information and basic attribute information (BOM) and product model information (document information) [4]. Product structure and attribute data which is derived by CAD system interface is imported to PDM product attribute database through the PDM system API function in XML format. Bidirectional integration between PDM and CAD can access product assembly model of CAD system through the API function of CAD system, and obtain product assembly relation, structure and parts information, etc, and then submit PDM product database through the PDM system API. Vice versa, it can derive product BOM information and model document through the API function of PDM system. CAD system reads product structure information and model information which is composed of its own API function. Finally construct product structure tree, update the product assembly model in CAD system. Fig.2 is the PDM and CAD two-way integrated structure.

\section{The integration research between Pro/E and Teamcenter}

In all kinds of information integration between CAX and PDM, especially the integration between CAD and PDM is the basic, key and most difficult technology. The study takes the current relatively popular CAD software Pro/E and PDM products Teamcenter information integration as 
an example, and discusses the realization of CAD and PDM information integration principle structure and key technology [5].

The integrated architecture. Generally speaking, application system information integration which is taken PDM as the platform mainly has application package, interface exchange and tightly integrated. In the implementation of integration, enterprise should be based on its technical level and actual application needs to select the integration mode, so as to allocate resources reasonably and to achieve the better effect. For the characteristic of CAD and PDM information exchange, combining the practical situation of businesses, the research uses interface exchange ways to conduct information integration in the form of data between Pro/E and Teamcenter. The integrated architecture between Pro/E and Teamcenter is shown in fig.3.

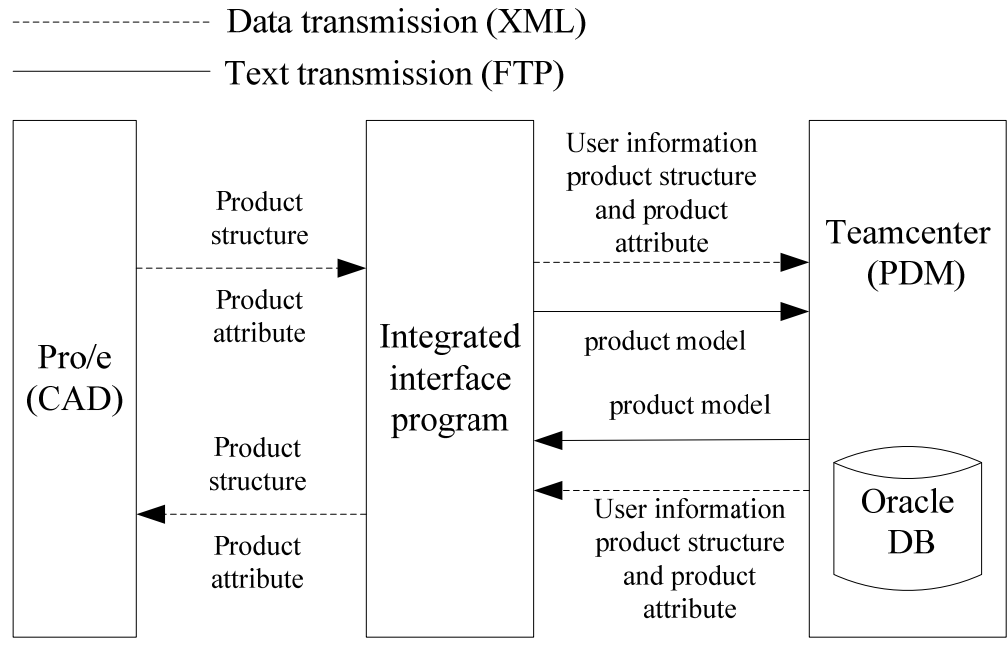

Fig.3 The integrated architecture between Pro/E and Teamcenter

The way of interface takes API and its function as the foundation, shielding the differences among integration system internal, also providing a guarantee for the interface programming smoothly and reliably. Therefore, this technology is applied widely in CAD and PDM integrated field, and it is especially suitable for Pro/E and Teamcenter integration.

The application case in enterprise. Based on the research, SAIC Information Company develops a set of Pro/E and Teamcenter integrated interface program, and get the practical application in enterprise. The interface is developed to satisfy the enterprise application, and its operation is simple and reliable. Save the model from Pro/E save to Teamcenter interface. Fig. 4 is the update interface of Teamcenter, the user can enter condition to inquire the model file. The inquired model document can browsed, checked out, downloaded and canceled according to the state in the dialog box. And then select the model to update.

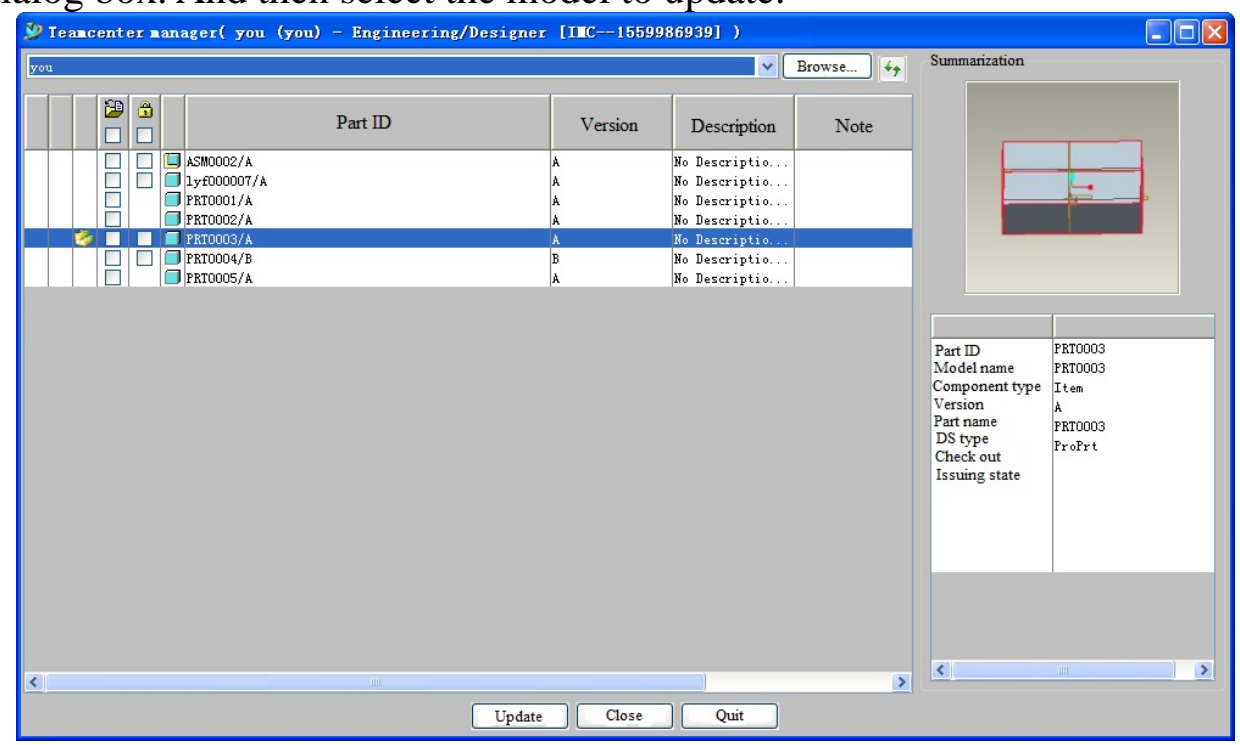

Fig.4 The update interface of Teamcenter 


\section{Summary}

PDM technology has made great progress since it was presented. All of the implementation technology, software development, integration and future and so on have a very broad space for development. Enterprise must to understand that information has turned from the agency to consultant, technical service and system integration era. However, the new technology has promoted the productivity development, and at the same time it brings new challenges. How to ensure the consistency from product data to the production and to avoid the data error and data redundancy has become the important demand of enterprise. The complete promotion and implementation of Teamcenter software is of great practical value to the company's document management, product structure, work process, parts classification, project management in the future, which will meet the needs of the development in enterprise.

\section{References}

[1] N.C. Do, G.S. Chae. A Product Data Management architecture for integrating hardware and software development. Computers in Industry 2011; 62(8-9): 854-863.

[2] J. Gao, D.T. Zheng, G. Nabil. Mathematical representation of feature conversion for CAD/CAM system integration. Robotics and Computer-Integrated Manufacturing 2004; 20(5): 457-467..

[3] N.W. Bowland, J.X. Gao, R. Sharma. A PDM and CAD integrated assembly modelling environment for manufacturing planning. Materials Processing Technology 2003; 138(3): 82-88.

[4] G.L. Ji, D.X. Gong, F. Tsui. Analysis and implementation of the BOM of a tree-type structure in MRPII. Journal of Materials Processing Technology 2003; 139(1-3): 535-538.

[5] H.S. Park, X.P. Dang. Structural optimization based on CAD-CAE integration and metamodeling techniques. Computer-Aided Design 2010; 42(10): 889-902. 